\title{
TRANSAÇÃO COM PARTE RELACIONADA COMO INSTRUMENTO DE FRAUDES CORPORATIVAS EM BANCOS BRASILEIROS
}

\author{
Júlio César Gomes Mendonça 1 \\ Michele Rílany Rodrigues Machado 2 \\ Ercilio Zanolla ${ }^{3}$ \\ José Alves Dantas 4
}

- Artigo recebido em: 26/03/2021 "- Artigo aceito em: 07/07/2021 ".- Segunda versão aceita em: 10/08/2021

\section{RESUMO}

Transações com partes relacionadas são transações que auditores, acionistas e acadêmicos temem que sejam utilizadas de maneira destoante do seu propósito econômico. A literatura considera transação com parte relacionada como uma proxy para a oportunidade de se cometer uma fraude. A fraude corporativa pode ser mais bem compreendida e analisada pelo triângulo de Cressey (1953). Este artigo tem por objetivo verificar se transações com partes relacionadas impactam na probabilidade de ocorrência de fraudes corporativas em instituições bancárias brasileiras de capital aberto. Para verificar a probabilidade da ocorrência de fraude foi estimada regressão logística com efeitos aleatórios, com dados de 24 bancos brasileiros de capital aberto, no período de 2010 ao segundo trimestre de 2019. Como principal resultado, verifica-se que transações com partes relacionadas aumentam a probabilidade de ocorrência de fraude corporativa, e o tipo de transação que se mostrou significante foi o propping. Conclui-se que transações passivas (propping) podem se dar de diversas

\footnotetext{
1 Mestre em Ciências Contábeis - UFG, Egresso Universidade Federal de Goiás, Universidade Federal de Goiás - Campus Samambaia. Endereço: R. Samambaia, s/n - Chácaras Califórnia, Goiânia - GO, 74001-970, Brasil. Telefone: (62) 3521-1390. E-mail: juliocgomesmend@gmail.com https://orcid.org/0000-0002-0263-9428
}

2 Doutora em Administração - UNB, Professora da Universidade Federal de Goiás - FACE/UFG, Universidade Federal de Goiás - Campus Samambaia. Endereço: R. Samambaia, s/n Chácaras Califórnia, Goiânia - GO, 74001-970, Brasil. Telefone: (62) 3521-1390. E-mail: michelemachado@ufg.br

https://orcid.org/0000-0002-1548-3071

${ }^{3}$ Doutor em Ciências Contábeis - UnB/UFPB/UFRN, Professor da Universidade Federal de Goiás FACE/UFG Universidade Federal de Goiás - Campus Samambaia. Endereço: R. Samambaia, s/n - Chácaras Califórnia, Goiânia - GO, 74001-970, Brasil. Telefone: (62) 3521-1390. E-mail: zanolla@ufg.br

https://orcid.org/0000-0002-7371-427X

${ }^{4}$ Doutor em Ciências Contábeis, Professor da Universidade de Brasília (UnB), Campus Darcy Ribeiro - Prédio da FACE. Endereço: Asa Norte, Brasília - DF, Brasília - DF, 70910-900, Brasil. Telefone: (61) 3107-0815. E-mail: josealvesdantas@unb.br https://orcid.org/0000-0002-0577-7340

Editora responsável pela aprovação do artigo: $\mathrm{Dr}^{\mathrm{a}}$. Bruna Camargos Avelino Editora responsável pela edição do artigo: $\mathrm{Dr}^{\mathrm{a}}$. Bruna Camargos Avelino 
maneiras ou motivos, mas não se deve considerar que todas as transações sejam realizadas para propósitos fraudulentos. O resultado do trabalho dá uma indicação de haver um aumento da probabilidade na ocorrência de fraude nas empresas estudadas. As preocupações dos auditores são válidas, e o estudo contribui para pesquisas sobre a importância e o impacto das partes relacionadas nas entidades.

Palavras-Chave: Transação com parte relacionada; Fraude corporativa; propping; tunneling.

\title{
RELATED-PARTY TRANSACTIONS AS AN INSTRUMENT OF CORPORATE FRAUD IN BRAZILIAN BANKS
}

\begin{abstract}
Related-party transactions are transactions that auditors, shareholders and academics fear may be carried out in a way that diverges from their true financial purpose. The literature regards related-party transactions as a proxy for an opportunity to commit fraud. Corporate fraud can be best understood when analyzed by the Cressey Fraud Triangle (1953). The purpose of this article is to determine whether related-party transactions have an effect on the likelihood of corporative fraud occurring in publicly-traded Brazilian banking institutions. This was determined through an estimate of logistic random effects regression based on data from 24 publicly-traded Brazilian banks in the period from 2010 to the second quarter of 2019. Our main result confirmed that related-party transactions make it more probable that corporate fraud will occur and the type of transaction that proved to be significant was propping [proprietary ("prop") trading]. It can be concluded that "propping" transactions (involving liabilities) can take place in several ways or for various reasons and it should not be assumed that all the transactions are carried out for fraudulent purposes. The results of the study suggest that that there is an increased likelihood of fraud in the companies studied. The concerns of the auditors are justified and this study makes a research contribution on the impact made by the related-party transactions on the entities.
\end{abstract}

Keywords: Related Party Transactions. Corporative Fraud. Propping. Tunneling.

\section{INTRODUÇÃO}

O setor bancário é de extrema importância para o funcionamento da economia, por disponibilizar créditos, movimentação de capital e operações financeiras. Deve-se lembrar que as falências de corporações bancárias podem levar a crises econômicas, como ocorreu com a quebra do Lehman Brothers em 2007/2008, um marco da chamada crise do subprime. Casos de fraudes envolvendo bancos e partes relacionadas acontecem como, por exemplo, o caso do Panamericano (2010). O banco fraudou seus resultados, se envolveu em diversas transações com organizações do mesmo grupo empresarial e realizou venda de ativos financeiros, sem o consequente registro das baixas no balanço, alterando seus indicadores econômico-financeiros, divulgando lucros irreais, gerenciando resultados com intuito fraudulento (Borges \& Andrade, 2018). 
Além do risco de fraudes praticados pelos próprios bancos, essas entidades também são utilizadas em processos fraudulentos envolvendo esquemas de desvios e lavagem de dinheiro. Exemplo nesse sentido pode se depreender de problemas identificados no âmbito da operação lava jato, iniciada em março de 2014, que descobriu diversos esquemas de corrupção envolvendo empresas privadas e públicas. A sofisticação do esquema envolveu a aquisição, pela Odebrecht, do Meinl Bank Antígua para realizar pagamentos de propinas, caracterizando uma transação com parte relacionada por motivos fraudulentos $(G 1,2016)$. Outro exemplo ilustrativo é o caso da fraude da Adelphia (EUA), em 2002, no qual os sócios proprietários a utilizavam como seu banco pessoal.

As fraudes corporativas, como as identificadas, podem ser analisadas pela teoria de fraudes, em especial pelo triângulo de fraudes proposto por Cressey (1953). Para Schuchter e Levi (2014), a teoria proposta relata que para a existência de atos fraudulentos é necessário existirem três elementos: (i) a pressão - quando o gestor considera que este é o único meio para resolver o seu problema; (ii) a moral - o gestor não encontra empecilhos morais para a realização; e (iii) a oportunidade - ocasião certa e ambiente propício para cometer o crime. Independentemente das motivações, é necessária a oportunidade para que a fraude ocorra.

Particularmente quanto à relação entre fraudes e as transações com partes relacionadas, Abdullahi e Mansor (2015) demostram que transação com parte relacionada é uma proxy para mensurar a oportunidade de se cometer uma fraude, ressaltando a frequência dessas transações nos casos de fraudes. Cohen, Ding, Lesage e Stolowy (2010), ao analisarem 39 casos de fraude de 1992 a 2005, constataram que 8 deles envolviam transações com partes relacionadas. Assim, indicam que este tipo de transação pode ser utilizado como mecanismo para fraudar as informações contábeis. Kohlbeck e Mayhew (2016) identificam que esse tipo de transação é um alerta para fraudes e está associada com a republicação das divulgações contábeis. Assim, tais transações podem ser utilizadas para fraudar ou gerenciar resultados e ainda podem estar associadas à republicação das divulgações.

Diante do exposto, tendo em vista a importância do setor bancário para a economia, bem como a carência de estudos que analisem a relação entre partes relacionadas e fraudes corporativas, é possível identificar uma problemática em comum: partes relacionadas, tidas como mecanismos de gerenciamento de resultados, influenciam na ocorrência de fraudes corporativas em instituições bancárias brasileiras de capital aberto?

O trabalho tem como objetivo verificar se transações com partes relacionadas, categorizadas como do tipo propping ou tunneling, impactam na probabilidade de ocorrência de fraudes corporativas em instituições bancárias brasileiras de capital aberto. A condição de propping, é definida por Friedman et al. (2003) como a condição em que a controlada transfere a riqueza para a controladora; o tunneling é a ação inversa, quando a controladora transfere riqueza para a controlada, conforme Pizzo (2011).

O trabalho se justifica visto que, geralmente, trabalhos sobre fraudes no Brasil optam por retirar as empresas financeiras do estudo (Borges \& Andrade, 2018), e por haver poucos estudos empíricos que buscam evidenciar a influência das transações com partes relacionadas na ocorrência de fraude. A pesquisa 
sobre parte relacionada é comentada por El-Helaly (2018), ao mencionar que a falta de dados disponíveis de países fora da Ásia é um desafio para a literatura sobre o tema, que provavelmente pode afetar o interesse dos pesquisadores sobre este campo de estudo. O presente trabalho colabora com 0 preenchimento desta lacuna de pesquisa e de forma adicional contribui para a discussão sobre a ocorrência de fraudes. O estudo ainda testa, empiricamente, se a preocupação dos pesquisadores, auditores e normatizadores com a utilização desta transação ocorrer de forma nociva e, por fim, auxilia os normalizadores ao verificar se a regulação vigente está sendo efetiva no combate ao mau uso deste tipo de transação.

O trabalho apresenta a seguinte estrutura: discussão do referencial teórico, abarcando o triângulo de fraude, transação com parte relacionada e fraudes corporativas; em seguida são apresentadas a metodologia de estudo e a análise dos resultados; por fim, a conclusão de pesquisa.

\section{REVISÃO DA LITERATURA}

O referencial teórico trata sobre o triângulo de fraude. O subtópico seguinte discorre sobre fraudes corporativas, realizando ligação entre a ocorrência de fraude e a transação com partes relacionadas. Por fim é apresentada a hipótese de pesquisa.

\subsection{Triângulo de Fraude}

Para Pedneault (2009) fraude é um conceito que possui diversas respostas dependendo do indivíduo e de seus valores. Todavia, para o presente trabalho, a definição de fraude segue o conceito de Coenen (2008), que a define como uma representação intencionalmente falsa sobre um ponto importante que faz com que a vítima sofra danos, quando alguém mente sobre um fato importante e outra pessoa perde dinheiro por causa disso. A maioria das fraudes é de fácil comprovação, pois geralmente é claro quando algo é falso e se há perda para alguém.

Já a fraude corporativa é a realizada contra ou a favor da companhia (Singleton, Singleton, Bologna e Lindquist, 2006) e pode ser classificada em dois tipos: (i) fraude diretamente contra a empresa - a empresa é a vítima e o funcionário é o beneficiado. Como exemplo, cite-se um desvio de fundos da empresa, fornecedores, clientes e outros subornando o funcionário; e (ii) fraude que beneficia a empresa - evasão fiscal, violação de leis ambientais e manipulação dos dados contábeis. Para Pedneault (2009) os esquemas comuns de fraude incluem reconhecimento prematuro de receita, capitalização de custos operacionais, falha em registrar ou divulgar passivos, entidades de interesse especial, estoques fictícios, custo reconhecido indevidamente e transações com partes relacionadas.

Pelo exposto, depreende-se que o processo de fraude é complexo. Para melhor compreensão do surgimento de fraude, Cressey (1953), sugeriu o triângulo de fraude que pressupõe que há necessidade de três elementos para ocorrer a fraude: a pressão, a racionalidade e a oportunidade. O triângulo de fraude foi utilizado como base teórica para diversos trabalhos para desenvolver e 
categorizar o risco de fraude (Schuchter\& Levi, 2014). A Figura 1 a seguir ilustra o triângulo.

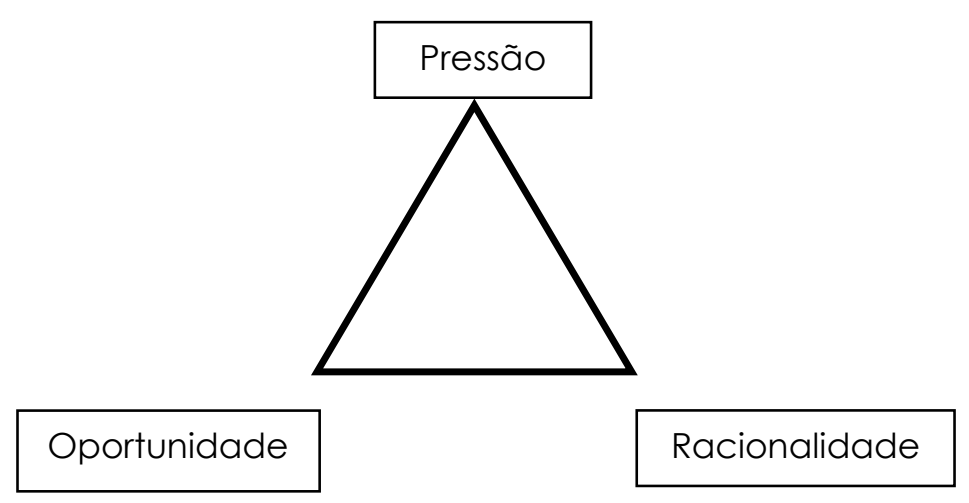

Figura 1 Triângulo de Fraude.

Fonte: Pedneault (2009, p.55).

A pressão ocorre quando o fraudador se sente pressionado e acredita que a única solução para o problema é cometer a fraude. Para Abdullahi e Mansor (2015) a pressão pode vir por motivos financeiros, não financeiros, políticos e sociais. Cressey (1953) discute diversos casos de fraude e traz o ponto de vista do fraudador e as justificativas resumidas pelo triângulo de fraude para explicar o ato ilícito. Todos os casos discutidos pelo autor têm um aspecto em comum: um problema financeiro a ser resolvido e o único meio de solução seria realizar o ato ilícito. São problemas não compartilháveis por conta dos quais a pessoa se sente pressionada a cometer o ato ilícito.

A racionalização é uma concepção do fraudador em formular alguma justificava moral, que seja aceitável, para cometer a fraude. Para Abdullahi e Mansor (2015) a racionalização se refere a criar justificativas e desculpas para o ato imoral. Para Cressey (1953) a pessoa de confiança financeira precisa justificar o seu ato, passando então para a verbalização, justificando, após o fato, a razão de ter cometido o ato fraudulento.

A oportunidade, para Abdullahi e Mansor (2015), é criada através do controle ineficiente ou do sistema de governança que permite o indivíduo cometer a fraude contra a organização. Para Coenem (2008) a oportunidade vem quando a empresa dá acesso ao ativo, às pessoas, à informação, ou seja, o fraudador costuma ser uma pessoa que possui alguma posição-chave na empresa, uma situação de poder. Cressey (1953) considera isto a oportunidade: é dada a uma pessoa confiável que, usando da sua posição, realiza o ato ilícito.

\subsection{Transação com Parte Relacionada e Fraude Corporativa}

No Brasil, o Conselho Monetário Nacional (CMN), por meio da Resolução nº 3.750, de 2009, impôs que as instituições autorizadas a funcionar pelo BCB deveriam divulgar informações sobre partes relacionadas conforme 0 Pronunciamento técnico CPC 05 - Divulgação de Partes Relacionadas, correlato à International Accounting Standard (IAS) 24. Em 2020, por meio da Resolução $C M N n^{\circ} 4.818$, foi promovida a atualização da norma, definindo que a partir de janeiro de 2021 as instituições financeiras passassem a observar os critérios do CPC 
05 (R1) na divulgação das transações com partes relacionadas. Essa normatização permitiu que a partir de 2009/2010 a divulgação das transações com partes relacionadas por parte das instituições bancárias brasileiras observasse os padrões disciplinados pelo International Accounting Standards Board (IASB) e pelo CPC, não obstante a defasagem na recepção da versão revisada (R1) do CPC 05. Em relação à associação entre transações com partes relacionadas (TPR) e a ocorrência de fraudes, tais eventos podem estar ligados à dimensão da oportunidade do triângulo de fraudes de Cressey (1953) devido à sua capacidade de expropriação, sem independência, além da ocorrência expost, isto é, só é percebida após o evento, como explicam Cheunget et al. (2006). Essas características de tais transações as tornam meio para a ocorrência da fraude, como no caso da Enron.

Shirur (2011) expõe a importância de se tomar cuidado com as TPR ao analisar os casos Enron e Satyam, em que a Enron é a retratação do problema de agência, enquanto o caso Satyam caracteriza o funneling. O autor argumenta que banir este tipo de transação seria impossível, tendo em vista haver várias situações em que esse tipo de transação gera benefícios econômicos aos acionistas, considerando a potencial sinergia entre as partes. De qualquer forma, esses casos ilustram o potencial de as TPR poderem ser utilizadas como meio de realização de fraude. Abdullahi e Mansor (2015) defendem que transação com parte relacionada é utilizada como proxy para a oportunidade. Koholbeck e Mayhew (2016) e Mangala e Kumari (2016) corroboram a percepção e o entendimento dos auditores, de que este tipo de transação, possa ser utilizado para expropriação e, assim, é motivo de preocupação. O trabalho de Koholbekc e Mayhew (2016) expõe que empresas que realizam TPR tendem a republicar seus balanços com maior frequência e as aquelas que realizam mais TPR tendem a pagar mais à auditoria. Para os autores, esses procedimentos são evidências de que os auditores reconhecem o risco das transações com partes relacionadas.

Estudos identificam consequências negativas ao realizar transações com partes relacionadas, mas El-Helaly (2018) percebe que nem todos os autores concordam que estas transações são sempre negativas, ou são indicativas para a realização de fraudes, e há evidências de que, para diferentes casos e contextos, há incidências de transações que foram vantajosas para as companhias.

O caso Enron, de 2001, ainda pode ser utilizado como o maior exemplo do potencial nocivo das transações com partes relacionadas. Para tornar a empresa mais atrativa para as agências de classificação de risco e os investidores, a empresa se empreendeu na utilização de entidades de propósito específico (SPE). A Enron contribuía com ativos tangíveis e uma dívida relacionada para a SPE e em troca recebia a participação. Então a SPE conseguia empréstimos para comprar ativos ou manter outros negócios e, assim, a dívida ou ativo não apareceriam na demonstração financeira consolidada da Enron evidenciando o maior retorno do ativo (ROA) e reduzindo o endividamento, a razão entre ativo total e passivo total (Thomas, 2002).

Instituições bancárias e financeiras também podem utilizar transações com partes relacionadas na realização de fraudes. Oda (2011) apresenta o acontecimento da Agrenco (2008), no qual a empresa de um dos conselheiros prestou serviços de assessoria na distribuição pública dos Brazilian Depositary Receipts (BDR). O banco de investimento que coordenou a operação possuía 200 Revista Contabilidade Vista \& Revista, ISSN 0103-734X, Universidade Federal de Minas Gerais, Belo Horizonte, v. 32, n. 3, p. 195-216, set./dez. 2021 
apenas um único analista para fazer a cobertura das ações da empresa e trabalhava para a companhia. Ou na Itália, na fusão da Fonsai e Unipol em 2012, quando o plano da Unifol era utilizar transações com partes relacionadas para integrar o mercado de seguro da Fonsai, mesmo havendo diversos conflitos de interesse. A situação foi crítica, e o presidente do comitê de partes relacionadas pediu demissão devido à falta da evidenciação das transações.

Giroux (2008), em sua análise dos escândalos e as lições que devem ser tomadas, demonstra que há dois motivos que incentivam a fraude entre partes relacionadas, a remuneração do executivo e alcançar os valores alvos dos analistas. Há, ainda, preocupação com os bancos de investimentos, nos quais existe um incentivo para o gerenciamento de resultado e a realização de fraudes. Os escândalos demonstram o encorajamento em investir em empresas relacionadas, deslocando o valor da ação de forma artificial.

Jiang, Lee e Yue (2010) verificam que, para as transações com parte relacionada na China, os empréstimos corporativos são vastos e são usados pelo acionista controlador para extrair fundos das empresas. Esses empréstimos representam uma porção significativa do ativo e capitalização do mercado chinês, e as transações são usadas intensivamente para transferir fundos. Empresas que possuem altos valores desses empréstimos são as que no futuro tendem a ter menor desempenho, sendo as candidatas a serem desalistadas do mercado acionário.

Os casos de fraude envolvendo transação com parte relacionada, para Pozzoli e Venuti (2014) podem levar a situação em que a sociedade em geral tenha a impressão de que a transação com parte relacionada são mais frequentes do que podem realmente ser. Assim, são necessários estudos para refutar ou aceitar esta impressão.

O caso do escândalo de Goldenberg (1993) é uma boa ilustração do uso de transação com parte relacionada para realização de fraude. O caso foi explicado por Akelola (2012) e ocorreu no Quênia. As empresas Goldenberg International Limited e Exchange Bank Limited tinham em comum os acionistas, promotores e diretores. O governo do Quênia dava incentivos à exportação de ouro e diamantes, e então se comprava ouro do Congo e se exportava como se fosse do Quênia, aproveitando-se, assim, dos incentivos. Essas operações eram realizadas pelo Exchange Bank Limited para compensação de exportação, conversão de moedas e outros serviços. Mesmo se passando alguns anos da descoberta, o escândalo ainda afetava o Quênia. O banco foi utilizado como meio operacional para a fraude.

Escândalos de fraude no Brasil e no mundo, como os da Enron, da Satyam e do Banco Panamericano envolveram, em algum grau, transações com parte relacionada. Tecnicamente, desde que haja um diretor ou executivo envolvido há presença de parte relacionada. Borges e Andrade (2018) citam que, no Brasil, em relação aos casos de fraudes em instituições bancárias há diversos exemplos, como os do banco Bamerindus, Banestes, BVA, Econômico, Cruzeiro do Sul, Santos, Nacional, Morada, Panamericano e Schahin. Dentre esses exemplos, nos casos do banco Panamericano e do Cruzeiro do Sul estiveram presentes transações com partes relacionadas através do PCLD.

O caso Panamericano envolveu a empresa Panamericano administradora de cartões. O banco transferia suas dívidas em situação de inadimplência para 
a relacionada, transação que não era fiscalizada pelo Banco Central do Brasil $(G 1,2012)$. O Banco Cruzeiro do Sul, no período de 2007 a 2012 utilizou os negócios com o fundo administrado pela Verax. A fraude ocorreu pela não evidenciação das transações realizadas fora do valor de mercado. Houve ainda omissões nas divulgações envolvendo a empresa Patrimonial Maragato. Apesar de a fraude envolver transações não realizadas diretamente pela instituição bancária, houve a utilização de recursos e comissões para favorecer os gerentes (Oliveira, 2016). Os casos supracitados demonstram a utilização deste tipo de transação com o propósito fraudulento.

Em uma população composta por auditores e professores que atuam na área de identificação de fraudes bancárias ou que pesquisam nessa área, Borges e Andrade (2018) verificam que as maiores preocupações dos especialistas são para a fraca governança corporativa, presença de transação com parte relacionada, ambiente de alta competição/pressão por desempenho, contas classificadas como diversos, PCLD e baixa liquidez. O predomínio dos entrevistados $(90,48 \%)$ considera que há uma relação de transação com parte relacionada na ocorrência de fraude.

Kohlbeck e Mayhew (2004) e Lu (2017) verificam a associação de transação com parte relacionada com a remuneração dos executivos e encontram que a maior independência dos diretores está associada ao baixo uso de TPR. Para Lu (2017), uma remuneração apropriada melhora o controle das TPRs e sugere que alguns tipos de transações são mais apropriados para a expropriação.

A expropriação do acionista pode ocorrer por outros meios, como exposto por Berkman, Cole e Fu (2007). Os autores demonstram que há o uso de garantias de empréstimos de partes relacionadas para expropriar o acionista minoritário. As empresas menores, mais lucrativas ou com melhor expectativa de crescimento foram as que apresentaram menores garantias de empréstimo e o artigo prova algumas evidências da relação do tunneling, o valor da empresa e o desempenho financeiro.

Além da garantia, Cheung et al. (2006) mostram que a compra e a venda entre partes relacionadas podem ser um meio de expropriação do acionista, quando as empresas pagam pelo ativo valores superiores ao normal do mercado e o vende por valor menor a uma transação normal de mercado. Transação com parte relacionada possibilita diversas oportunidades para que os gestores cometam atos ilícitos.

No Brasil, Alves e Alves (2008) verificam a qualidade da divulgação contábil e constatam que a categoria empresas ligadas e partes relacionadas é a que apresenta o menor porcentual de divulgação (16,7\%). Essa baixa divulgação pode ser um indicativo do mau uso deste tipo de transação. Para Petrucelli (2012), no entanto, a divulgação inadequada não necessariamente significa dizer que estas informações sejam fraudulentas, entretanto é provável que sejam, como corrobora Wells (2011) ao afirmar que a não divulgação de transação com parte relacionada pode ser um sinal de alerta.

Uma expressão do português, "a ocasião faz o ladrão", é um velho ditado da sabedoria popular que considera que para que um ladrão aja é necessário que exista a oportunidade. Para Schuchter e Levi (2014), independentemente da motivação do gestor, para a realização de um ato delinquente é necessária a 
oportunidade. A literatura traz a relação teórica entre as partes relacionadas e a ocorrência de fraude. Bancos se situam em um ambiente complexo e regulado, e este pode ser um incentivador de comportamento oportunístico. Assim, apresenta-se a seguinte hipótese de pesquisa:

\section{$H_{1}$ : Há associação positiva entre o volume de transações com partes relacionadas e a probabilidade de ocorrência de fraudes corporativas em bancos brasileiros de capital aberto.}

\section{PROCEDIMENTOS METODOLÓGICOS}

Para a relação dos testes empíricos, com o propósito de verificar se há associação entre a probabilidade de ocorrência de fraudes e o nível de transações com partes relacionadas, este trabalho se utiliza de regressão logística, com a finalidade de testar a hipótese de pesquisa. Para tanto, nesta seção são especificados a população objeto de estudo, o período amostral, a forma de coleta de dados e o detalhamento do modelo e das variáveis utilizadas para as estimações estatísticas.

\subsection{População, Período e Coleta de Dados}

A população é composta pelos 24 bancos brasileiros de capital aberto, com registro na CVM, compreendendo dados trimestrais entre 2010 e o segundo trimestre de 2019, resultando em 864 observações. A opção em se restringir o período a partir de 2010 se justifica pelo fato de ter sido o momento de implementação da previsão de divulgação das transações com partes relacionadas, mitigando-se os efeitos relacionados à mudança dos critérios e requisitos de divulgação. Os dados das instituições bancárias foram obtidos no relatório IF.Data, diretamente na página do Banco Central do Brasil (BCB), na internet, durante o segundo semestre de 2019. Os tratamentos dos dados e as estimações estatísticas foram realizados com o uso do software STATA®.

\subsection{Variáveis e Regressão}

Para verificar se há associação entre transações com partes relacionadas e a ocorrência de fraudes, o trabalho utiliza a estatística descritiva (média, mediana, frequência, máximo e mínimo e desvio-padrão) e testes estatísticos (regressão logística) com a finalidade de responder à questão de pesquisa.

O presente trabalho utiliza como proxy para a variável dependente, ocorrência de fraude (OcorFr), de modo similar a Machado e Gartner (2018). Portanto, é uma variável dummyque assume valor 1 para empresas condenadas no Conselho de Recursos do Sistema Financeiro Nacional (CRSFN) e 0 para as demais. Esclarece-se que foram analisados os processos administrativos de instituições bancárias julgadas e condenadas pelo CRSFN durante o período da pesquisa. Após a leitura de cada Acórdão emitido pelo CRFN e das documentações disponíveis no processo, identificou-se como fraude apenas o período exposto na condenação como o da perpetração do ato fraudulento.

Frise-se que o CRSFN é o órgão responsável por julgar, em segunda instância, os processos administrativos punitivos do BCB. Assim, houve a divisão da 
amostra em dois grupos: com e sem fraudes corporativas. Reforce-se que a classificação segue o padrão de Machado e Gartner (2018), segundo os quais o conceito de fraude corporativa segue a definição de Singleton et al. (2006) e, deste modo, foram analisados os processos que envolvem a entidade - no caso, os bancos de capital aberto.

Para a mensuração das variáveis independentes de interesse foram coletadas: (i) transações com partes relacionadas, nas notas explicativas das demonstrações; (ii) ativo total e passivo total, no IF.Data do BCB; e (iii) nível de transações com partes relacionadas, conforme método utilizado por Supatmi et al. (2019), que define esse patamar em função dos conceitos de saída de recursos da matriz para a parte relacionada e de entrada de recursos da subsidiária para a matriz.

A saída é definida pela razão entre o total de contas a receber com partes relacionadas (CPR) dividida pelo ativo total (AT). Considerada como tunneling, o termo é definido por Shirur (2011) como sendo a transferência de ativos e lucros para fora da firma. O resultado desta equação é considerado para propósito do trabalho como nível das transações ativas (NPRA). Por outro lado, a entrada é definida como a razão entre o total das contas a pagar com partes relacionadas (RPR) dividida pelo passivo total (PT). Encarada como de natureza propping, o termo é definido por Friedman et al. (2003) como a condição na qual as partes relacionadas transferem riqueza para a controladora. O resultado desta equação é o nível de transação com parte relacionada passivas (NPRP). Esse conjunto de variáveis representativas do nível de transações com partes relacionadas (NPR) é destacado na equação (1):

$$
N P R_{i t}=\frac{C P R_{i t}}{A T_{i t}}-\frac{R P R_{i t}}{P T_{i t}} \text {, isto é: } N P R_{i t}=N P R A_{i t}-N P R P_{i t}
$$

Definidas as variáveis dependentes (OcorFr) e as independentes de interesse (NPR, NPRA e NPRP), resta definir as variáveis de controle que serão utilizadas pelo trabalho. As variáveis de controle são variáveis relacionadas ao tamanho, a relação macroeconômica e variáveis relacionadas à visão do triângulo de fraude (Pressão, Racionalização e Oportunidade).

Para as variáveis referentes à racionalização, devido a situações apontadas por Brazel, Jones e Zimbelman (2009), como há necessidade de mais recursos humanos e maior grau de conluio, portanto, de difícil mensuração e identificação, optou-se em não as utilizar. As variáveis do modelo em relação à oportunidade são a participação acionária do principal acionista e mudança de auditoria. Para o âmbito da pressão se utiliza o tamanho, mensurado pelo logaritmo natural do ativo total, prejuízo defasado, produto interno bruto do país e o retorno do ativo.

A Tabela 1 apresenta um sumário das variáveis dependente, de interesse e de controle utilizadas na estimação do modelo para identificação dos determinantes da ocorrência de fraudes. 
Tabela 1

Variáveis dependente, de interesse e de controle para estimar a ocorrência de fraudes

\begin{tabular}{|c|c|c|c|c|c|}
\hline Variável & Dimensão & Fórmula & Sinal & Fonte & Autores \\
\hline \multicolumn{6}{|c|}{ Variável Dependente } \\
\hline $\begin{array}{l}\text { Ocorfr } \\
\text { (Ocorrência } \\
\text { de fraude) }\end{array}$ & & $\begin{array}{l}\text { Variável dummy: } \\
1 \text { para empresas } \\
\text { condenadas no } \\
\text { CRSFN e } 0 \text { para } \\
\text { as demais. }\end{array}$ & & $\begin{array}{l}\text { Processos } \\
\text { administrativos } \\
\text { do CRSFN }\end{array}$ & $\begin{array}{l}\text { Machado } \\
\text { e Gartner } \\
\text { (2018) }\end{array}$ \\
\hline \multicolumn{6}{|c|}{ Variáveis de Interesse } \\
\hline $\begin{array}{l}\text { NPR (Nível de } \\
\text { TPR) }\end{array}$ & & $\begin{array}{l}\text { NPRA - NPRP } \\
\text { (definidas em } \\
\text { seguida) }\end{array}$ & & $\begin{array}{l}\text { Notas } \\
\text { explicativas e } \\
\text { IF.Data }\end{array}$ & $\begin{array}{l}\text { Supatmi et } \\
\text { al. }(2019)\end{array}$ \\
\hline $\begin{array}{l}\text { NPRA (Nível de } \\
\text { TPR ativa) }\end{array}$ & & $\begin{array}{l}\text { Relação entre os } \\
\text { créditos a } \\
\text { receber de } \\
\text { partes } \\
\text { relacionadas e o } \\
\text { ativo total }\end{array}$ & & $\begin{array}{l}\text { Notas } \\
\text { explicativas e } \\
\text { IF.Data }\end{array}$ & $\begin{array}{l}\text { Supatmi et } \\
\text { al. (2019) }\end{array}$ \\
\hline $\begin{array}{l}\text { NPRP Nível de } \\
\text { TPR passiva) }\end{array}$ & & $\begin{array}{l}\text { Relação entre as } \\
\text { contas a pagar a } \\
\text { partes } \\
\text { relacionadas e o } \\
\text { passivo total }\end{array}$ & & $\begin{array}{l}\text { Notas } \\
\text { explicativas e } \\
\text { IF.Data }\end{array}$ & $\begin{array}{l}\text { Supatmi et } \\
\text { al. }(2019)\end{array}$ \\
\hline \multicolumn{6}{|c|}{ Variáveis de Controle } \\
\hline $\begin{array}{l}\text { Own } \\
\text { (concentração } \\
\text { acionária) }\end{array}$ & Oportunidade & $\begin{array}{l}\text { Porcentagem de } \\
\text { participação } \\
\text { acionária do } \\
\text { maior acionista } \\
\text { (ações totais) }\end{array}$ & + & $\begin{array}{l}\text { Formulário de } \\
\text { referência }\end{array}$ & $\begin{array}{l}\text { Supatmi et } \\
\text { al. (2019) } \\
\text { Chen, Zhu } \\
\text { e Wang } \\
(2011)\end{array}$ \\
\hline $\begin{array}{l}\text { Tam (tamanho } \\
\text { da entidade) }\end{array}$ & Pressão & $\begin{array}{l}\text { Logaritmo natural } \\
\text { dos ativos totais }\end{array}$ & + & IF.Data & $\begin{array}{l}\text { Koholback } \\
\text { e Mayhew } \\
\text { (2010) } \\
\text { Supatmi et } \\
\text { al. (2019), } \\
\text { Chen et al. } \\
\text { (2011) Lou } \\
\text { e Wang } \\
\text { (2009) }\end{array}$ \\
\hline $\begin{array}{l}\text { dPrej } \\
\text { (ocorrência de } \\
\text { prejuízo) }\end{array}$ & Pressão & $\begin{array}{l}\text { Variável dummy: } \\
1 \text { para empresas } \\
\text { que } \\
\text { apresentaram } \\
\text { prejuízo no } \\
\text { período anterior } \\
\text { e } 0 \text { para outras }\end{array}$ & + & $\begin{array}{l}\text { Demonstrações } \\
\text { financeiras }\end{array}$ & $\begin{array}{l}\text { Firth, Rui e } \\
\text { Wu (2011) } \\
\text { Koholback } \\
\text { e Mayhew } \\
\text { (2010) Lou } \\
\text { e Wang } \\
\text { (2009) }\end{array}$ \\
\hline $\begin{array}{l}\text { ROA (retorno } \\
\text { sobre os ativos) }\end{array}$ & Pressão & $\begin{array}{l}\text { Relação entre o } \\
\text { lucro líquido e os } \\
\text { ativos totais do } \\
\text { final do período }\end{array}$ & - & IF.Data & $\begin{array}{l}\text { Koholback } \\
\text { e Mayhew } \\
\text { (2010) } \\
\text { Supatmi et } \\
\text { al. (2019), } \\
\text { Chen et al. } \\
\text { (2011) }\end{array}$ \\
\hline $\begin{array}{l}P I B \text { (produto } \\
\text { interno bruto) }\end{array}$ & Pressão & $\begin{array}{l}\text { Variação do PIB } \\
\text { no trimestre }\end{array}$ & - & IBGE & $\begin{array}{l}\text { Botinha \& } \\
\text { Leme } \\
(2019)\end{array}$ \\
\hline
\end{tabular}




\begin{tabular}{l|l|l|l|l|l}
\hline \multicolumn{1}{c|}{ Variável } & Dimensão & \multicolumn{1}{c|}{ Fórmula } & Sinal & \multicolumn{1}{c|}{ Fonte } & \multicolumn{1}{c}{ Autores } \\
\hline $\begin{array}{l}\text { MudAudit } \\
\text { (mudança do } \\
\text { auditor) }\end{array}$ & Oportunidade & $\begin{array}{l}\text { Variável dummy } \\
\text { em que 1 para } \\
\text { mudança de } \\
\text { auditor e 0, para } \\
\text { outras }\end{array}$ & + & $\begin{array}{l}\text { Notas } \\
\text { explicativas }\end{array}$ & $\begin{array}{l}\text { Skousen, } \\
\text { Smith e } \\
\text { Wright } \\
\text { (2009) }\end{array}$ \\
\hline
\end{tabular}

Fonte: Elaborada pelos autores.

A variável de governança (Own) é incorporada com a premissa de que a governança corporativa tem um grande impacto neste tipo de transação, servindo para mitigar o efeito nocivo e, portanto, se espera um sinal negativo para esta variável (Chen et al., 2011; Koholback \& Mayhew, 2010; Lo et al., 2010 e Supatmi et al., 2019). O sentido de se controlar por aspectos de governança se deve à natureza deste tipo de transação, em que o acionista majoritário pode lançar a mão das TPR para expropriar o minoritário e, portanto, se espera um sinal positivo para esta variável.

As outras variáveis de controle são para o tamanho e para o ambiente macroeconômico $(P / B)$. A variável Tam foi utilizada para controlar possíveis diferenças acarretadas pelo tamanho da empresa (Chen et al., 201 1, Koholback \& Mayhew, 2010 e Supatmi et al. 2019). Lou e Wang (2009) consideram que há uma forte associação entre o tamanho da empresa e a fraude, portanto se espera um sinal positivo para esta variável. A variável PIB foi utilizada para controlar o nível da atividade econômica e, considerando que a taxa de crescimento está associada à corrupção, em que baixos crescimentos econômicos há crescimento da ocorrência de fraudes, espera-se um sinal negativo (Botinha \& Lemes, 2019).

As variáveis $R O A$ e dPrej defasado são utilizadas para controlar empresas que podem estar sofrendo pressão financeira, situação para a qual os autores consideram que empresas apresentam prejuízo (Firth et al., 2011, Lou \& Wang, 2009 e Koholback \& Mayhew, 2010). Para o cálculo do ROA, foram utilizadas as contas do COSIF, conta de resultado credora (7.0.0.00.00-9) mais a devedora (8.0.0.00.00-6), dividido pelo somatório do ativo circulante e realizável a longo prazo (1.0.0.00.00-7) com o ativo permanente (2.0.0.00.00-4). A variável MdAudit é aplicada pelo fato de a literatura considerar que a incidência de falhas e litígios aumentam imediatamente após a mudança de auditoria, fato observado por Skousen et al. (2009). As variáveis de interesse NPR, NPRA e NPRP são utilizadas para verificar o nível das transações com partes relacionadas da empresa da amostra, para a qual se espera o sinal positivo (Supatmi et al., 2019).

A regressão utilizada foi a logística, portanto para a primeira estimação de parâmetros de um modelo logístico para dados em painel (PooledLogif) deve-se considerar a existência de erros-padrão robustos com agrupamento por indivíduo, a fim de que haja o controle da correlação dos termos de erro, porém o tempo é fixo e, devido a isso, não é o modelo mais adequado. Os parâmetros por efeitos fixos ou aleatórios são mais adequados que o PA logit, pois este modelo considera o coeficiente médio da população. Portanto o modelo probabilístico a ser estimado é o logit com efeitos aleatórios, por se tratar de pouca variabilidade de sujeitos, e as variáveis omitidas não estão correlacionadas com modelo (Allison, 2009). Assim, o modelo apresenta a seguinte expressão: 


$$
P(\text { OcorFr })_{i t}=\frac{\left.e^{\left(\alpha_{i}+\beta_{1} N P R_{i t}+\beta_{2} O w n_{i t}+\beta_{3} \text { Tam }_{i t}++\cdots+\beta_{6} \text { MudAudit }_{i t}\right.}\right)}{1+e^{\left(\alpha_{i}+\beta_{1} N P R_{i t}+\beta_{2} O w n_{i t}+\beta_{3} \text { Tam }_{i t}+\cdots+\beta_{6} \text { MudAudit }_{i t}\right)}}
$$

A situação da multicolinearidade foi verificada através da estatística VIF (Variance Inflation Factor), que se torna problemática quando há uma correlação elevada entre as variáveis explicativas. Para Fávero \& Belfiore (2017), há existência da multicolinearidade quando o valor do VIF é acima de 10.

\section{ANÁLISE E DISCUSSÃO DOS RESULTADOS}

Foram analisados os dados trimestrais dos bancos de capital aberto brasileiros, do período de 2010 ao segundo trimestre de 2019. Nesse período, uma entidade (HSBC) encerrou suas atividades, enquanto outra teve mudança do acionista controlador (Banco Industrial do Brasil) e passou a ser denominada China Construction Bank. Nesta última situação os bancos foram tratados como duas instituições diferentes, devido à mudança de suas partes relacionadas. A Tabela 2 apresenta as estatísticas descritivas das variáveis do trabalho.

\section{Tabela 2}

Estatísticas descritivas: variáveis de controle e nível de transações com partes relacionadas

\begin{tabular}{cccccccc} 
& NPR & NPRP & NPRA & ROA & Own & Tam & PIB \\
\hline No Observações & 848 & 847 & 848 & 848 & 852 & 848 & 875 \\
Mínimo & $-0,614$ & 0 & 0 & $-0,051$ & 7,3 & 14,352 & $-4,500$ \\
Máximo & 0,163 & 0,614 & 0,381 & 0,107 & 100 & 21,156 & 7,500 \\
Média & $-0,064$ & 0,097 & 0,032 & 0,0051 & 57,949 & 17,204 & 1,375 \\
Mediana & $-0,017$ & 0,035 & 0,001 & 0,005 & 55,75 & 16,535 & 1,400 \\
Desvio Padrão & 0,138 & 0,136 & 0,058 & 0,009 & 28,325 & 1,843 & 3,078 \\
\hline
\end{tabular}

Em que NPRé o nível de TPR; NPRA é o nível de TPR ativa; NPRPé o nível de TPR passiva; $R O A$ corresponde ao retorno sobre os ativos; Own é o grau de concentração acionária, representada pela participação dos cinco principais acionistas; Tam é a proxy de tamanho da entidade, mensurada pelo logaritmo natural dos ativos totais; $e$ PIB representa o nível de atividade econômica, representado pela variação do PIB.

Fonte: Dados de Pesquisa.

As variáveis representativas do nível de transações com partes relacionadas demonstram que, em média, há mais transações passivas do que ativas com a companhia, portanto, há mais transações do tipo propping do que tunneling. Assim, a empresa matriz recebe mais dinheiro de suas subsidiárias/relacionadas. Na amostra há algumas empresas que não apresentam transações ativas (tunneling) ou passivas (propping) com suas subsidiárias, nas notas explicativas da companhia não há menção da motivação da não existência deste tipo de transação no período. 
As estatísticas descritivas demonstram que o setor financeiro apresenta ROA médio de 0,005, o que é associado à característica de alta alavancagem inerente ao sistema bancário. Em relação ao controle, os bancos brasileiros de capital aberto apresentam apenas um controlador com mais de $50 \%$ das ações, assim, essa situação pode ser incentivadora de comportamento oportunístico. Para Geroux (2008), o gestor poderia ser encorajado a investir na parte relacionada com o intuito de melhorar o preço da ação. A variável PIB indica que em média o Brasil obteve um crescimento trimestral de 1,37\%, havendo períodos de baixa $(-4,5 \%)$ e alta de $(7,5 \%)$, sendo que os períodos de baixa podem ser momentos de incentivos ao mau comportamento do gestor.

Com relação às variáveis qualitativas à ocorrência de fraude, se verifica que em 7,55\% (64) da amostra houve a ocorrência de fraudes nas entidades analisadas. A ocorrência pode estar associada por considerar apenas os processos identificados com a denúncia e condenação. Para o prejuízo defasado, 11,38\% (99) da amostra apresentam prejuízo no período, a maior parte do período em análise os bancos brasileiros apresentam lucratividade. Com relação à mudança de auditoria, 11,76\% (100) da amostra apresentam mudança de auditoria, portanto se verifica certa estabilidade. 3.

O resultado da estimação da regressão logística é apresentado na Tabela 
Tabela 3

Regressão logística de ocorrência de fraude corporativa

\begin{tabular}{|c|c|c|c|c|c|c|}
\hline Variáveis & Fraude & OR & Fraude & OR & Fraude & OR \\
\hline$N P R$ & $\begin{array}{c}-9,60186^{*} \\
(-4,11)\end{array}$ & $0,00006^{*}$ & & & & \\
\hline NPRA & & & $\begin{array}{c}-5,02180 \\
(-1,48)\end{array}$ & 0,00659 & & \\
\hline NPRP & & & & & $\begin{array}{c}9,05634^{*} \\
(3,90)\end{array}$ & 8572,789 \\
\hline Own & $\begin{array}{c}0,081144^{*} \\
(2,98)\end{array}$ & $1,08452^{*}$ & $\begin{array}{c}0,04340^{* * *} \\
(1,93)\end{array}$ & $1,04435^{* * *}$ & $\begin{array}{c}0,06648^{*} \\
(3,17)\end{array}$ & $1,06874^{*}$ \\
\hline Tam & $\begin{array}{c}-3,13793^{*} \\
(-2,76)\end{array}$ & $0,04337^{*}$ & $\begin{array}{c}-0,81028 \\
(-1,01)\end{array}$ & 0,44473 & $\begin{array}{c}-2,61755^{*} \\
(-3,83)\end{array}$ & 0,07298 \\
\hline dPrej & $\begin{array}{c}-0,05618 \\
(-0,09)\end{array}$ & 0,94536 & $\begin{array}{c}0,20760 \\
(0,35)\end{array}$ & 1,23072 & $\begin{array}{c}0,25345 \\
(0,43)\end{array}$ & 1,28847 \\
\hline$R O A$ & $-54,36284$ & $2,46 e-24$ & $-33,25707$ & $3,60 \mathrm{e}-15$ & 46,69214 & $5,27 e-21$ \\
\hline & $(-1,35)$ & & $(0,35)$ & & $(-1,23)$ & \\
\hline$P / B$ & $\begin{array}{c}0,26005^{* *} \\
(2,41)\end{array}$ & $1,297^{* *}$ & $\begin{array}{c}0,19086^{* *} \\
(2,03)\end{array}$ & 1,21030 & $\begin{array}{c}0,32116^{*} \\
(3,04)\end{array}$ & 1,37873 \\
\hline MudAudit & $\begin{array}{c}3,08126 \\
(1.38)\end{array}$ & 21,78595 & $\begin{array}{c}2,38192 \\
(1,52)\end{array}$ & 10,82572 & $\begin{array}{c}2,81473 \\
(1,51)\end{array}$ & 16,68868 \\
\hline Cons & $\begin{array}{c}18,91821 \\
(1,10)\end{array}$ & $1,64 e+08$ & $\begin{array}{c}-3,20383 \\
(-0,25)\end{array}$ & 0,04060 & $\begin{array}{c}17,24482 \\
(1,52)\end{array}$ & $3,09 e+07$ \\
\hline
\end{tabular}

Em que OR é a razão de chance; NPRé o nível de TPR; NPRA é o nível de TPR ativa; NPRPé o nível de TPR passiva; ROA corresponde ao retorno sobre os ativos; Own é o grau de concentração acionária, representada pela participação dos cinco principais acionistas; Tamé a proxy de tamanho da entidade, mensurada pelo logaritmo natural dos ativos totais; dPrejé uma variável dummy indicativa da ocorrência de prejuízo no período anterior; PIB representa o nível de atividade econômica, representado pela variação do PIB; e MudAudit é uma variável dummy representativa da ocorrência de alteração nos auditores responsáveis por emitir opinião sobre as demonstrações financeiras.

${ }^{*} p<0,01,{ }^{* *} p<0,05, p<0,1$. ZScore em parênteses.

Fonte: Dados de pesquisa 
A regressão utilizada foi painel com efeitos aleatórios e foi testado se há problemas de multicolinearidade na base de dados. Foi verificado um VIF médio de 1,16. Para correção de possíveis problemas de multicolinearidade e heterocedasticidade, a variável Tam foi transformada em seu logaritmo natural. Não foram identificados problemas com outliers.

Na regressão logística em questão, o coeficiente da variável indica a probabilidade de ocorrência de fraude. Deste modo, o coeficiente positivo indica um aumento na tendência de ocorrência de fraude e o sinal negativo, uma redução. Assim, quando o NPR é estatisticamente significante com coeficiente positivo, aceita-se a hipótese de que transações com partes relacionadas aumenta a probabilidade da ocorrência de fraude, resultado corroborado pela percepção de Abudullahi e Mansor (2015), Mangala e Kumari (2016) e Koholbeck e Mayhew (2016). Seguindo, assim, a literatura sobre o tema, dentro do triângulo de fraude, essas transações podem ser uma proxy de oportunidade de fraude.

Ao analisar as variáveis NPRA e NPRP, se verifica qual tipo de transação (ativa ou passiva) possa estar aumentando ou diminuindo a probabilidade de ocorrência de fraude. O resultado indica que a variável NPRP é positiva e com significância estatística. Assim, nas transações nas quais o banco gera um passivo com sua relacionada (propping) aumenta a probabilidade na ocorrência de fraude (o aumento de um ponto no nível de transações passivas aumenta a probabilidade de ocorrência de fraude em 8572,789\%), resultado similar ao de Jiang, Lee e Yue (2010) transação com parte relacionada pode estar sendo utilizada para transferir fundos. O aumento da probabilidade ser maior do que 0 aumento da transação com parte relacionada ativa $(0,06 \%)$, pode ser um indicativo onde apenas transações passivas são relevantes na fraude. Não se rejeita a hipótese de pesquisa, de que transações com partes relacionadas têm associação positiva com a probabilidade de ocorrência de fraudes corporativas em bancos brasileiros de capital aberto e, como resultado adicional, as transações utilizadas são passivas (propping).

Propping são as transações em que a controladora possui algum direito econômico sobre a controlada, seja contratação de serviços, empréstimos, compra de bens dentre outras transações. A base de dados não permite inferir qual tipo de transação passiva possa estar influenciando, mas já se verifica a influência deste tipo de transação. A mesma limitação na base de dados pode ser mencionada para as transações ativas (Tunneling). Não é possível indicar qual tipo de transação esteja influenciando de forma positiva/negativa a probabilidade de ocorrência de fraude, mas há uma indicação de que a controladora, ao ser contratada pela parte relacionada, empréstimos e demais transações possuem menor impacto na probabilidade de fraude.

A variável relativa ao controle por parte do principal acionista (Own) se mostrou significante para os três modelos. O resultado indica que, quanto maior - controle acionário maior a probabilidade de ocorrência de fraude, as probabilidades nos três modelos são similares (108\%, 104\% e 106\%), reforçando a noção que quanto maior o controle maior a probabilidade de ocorrência de fraudes. Esse resultado pode estar associado ao motivo remuneratório destacado por Koholbeck e Mayhew (2004). O controle mais concentrado da entidade pode estar diminuindo a independência dos diretores e, assim, o acionista majoritário pode estar utilizando este tipo de transação como oportunidade para cometer 
fraude. Lu (2017) considera que alguns tipos de transações são mais apropriados para a fraude, e o resultado para a amostra analisada identifica que as transações passivas (propping) impactam na probabilidade da ocorrência de fraude.

Quanto ao tamanho, este se mostrou significante para NPR e NPRP. O tamanho da empresa reduz a tendência da ocorrência da fraude, e o resultado é contrário ao de Lo e Wang (2009). Os autores consideram que empresas maiores são sujeitas a fraudes, mas para os bancos de capital aberto se espera que, devido à sua importância econômica e social, tendem a sofrer controles mais rígidos, inibindo assim ações prejudiciais ao acionista minoritário. Entretanto, para - modelo com as transações ativas a probabilidade do tamanho (44\%) se mostrou superior aos outros dois modelos (Nível da transação (4\%) e passiva 7\%), e este resultado pode estar relacionado ao volume, em que para empresas maiores espera-se que possuam maior frequência em transação com as subsidiárias. O prejuízo defasado e rentabilidade, $R O A$, não apresentaram significância estatística e, assim, não impactam na probabilidade de ocorrência de fraude.

Já para o indicador econômico, PIB, se verifica a significância estatística para os três modelos, em situação de crescimento econômico, e é mais suscetível a ocorrência de fraudes (a probabilidade encontrada é superior a 1), o resultado vai ao encontro ao trabalho de Botinha \& Lemes (2019), para o qual o crescimento econômico está associado à fraude. Seguindo, assim, o mesmo resultado para as outras variáveis, o tipo de transação que possui maior tendência de ser fraude são transações passivas (propping), dando maior robustez para a visão de que bancos de capital aberto possam estar utilizando transações com partes relacionadas passivas com propósito escuso. As variáveis de pressão do triângulo de fraude (Tam, dPrej, ROA e PIB), conseguiram captar pressões socioeconômicas nos bancos que impactam a ocorrência de fraude, este resultado pode ser uma indicação que dentro do estudo de fraude, as instituições bancárias são mais suscetíveis a pressões para a realização da fraude.

Por fim, para a variável relativa à mudança da auditoria (MudAudif) não apresentou significância estatística e, portanto, a mudança da auditoria não afeta a probabilidade de ocorrência de fraude bancária, resultados que são contrários à literatura, como os trabalhos de Machado (2015), Oda (2011) e Skousen et al. (2009). Em decorrência da limitação da base, não é possivel assumir a razão deste resultado diferente, podendo ser por aspectos regulatórios, sociais, temporais e econômicos. No modelo a mudança de auditoria não se provou capaz de captar o aspecto da oportunidade no triângulo de fraude.

\section{CONCLUSÕES}

A pesquisa buscou analisar se a preocupação dos auditores demostrada por Borges e Andrade (2018) é válida, de que transações com partes relacionadas possam estar associadas à ocorrência de fraude. Para isso utilizouse a regressão logística para verificar a confirmação da hipótese de pesquisa, de que partes relacionadas impactam positivamente a probabilidade de ocorrência de fraudes corporativas em bancos brasileiros de capital aberto. Os resultados 
confirmam a hipótese, nos casos de operações passivas, caracterizando operações de propping.

O triângulo de fraude é um dos métodos que buscam racionalizar as motivações da existência da fraude, incluindo esse tipo de transações, e, considerando a afirmação de Shirur (2011) de que é irracional banir este tipo de transação com parte relacionada, torna-se necessário compreender os seus impactos de forma objetiva. Nessa análise racional, além do efeito econômico, de governança corporativa e do impacto societário, tais eventos podem impactar a ocorrência de fraude, como demonstram as evidências empíricas.

Por impactar na probabilidade na ocorrência de fraude, transações com partes relacionadas podem ser utilizadas como proxy para a oportunidade de cometer uma fraude, seguindo a perspectiva de Abdullahi e Mansor (2015), Mangala e Kumari (2016) e Koholbeck e Mayhew (2016). Como resultado adicional, a pesquisa identifica que as transações passivas (propping) são as que aumentam a tendência da ocorrência de fraude, enquanto que para as ativas (tunneling) não foi identificada associação estatística.

Transações passivas (propping) podem se dar de diversas maneiras ou motivos, mas não se deve considerar que todas as transações sejam realizadas para propósitos fraudulentos (Pozzoli\&Venuti, 2014). O resultado do trabalho dá uma indicação de haver um aumento da probabilidade na ocorrência de fraude nas empresas estudadas. Assim, as preocupações dos auditores apresentadas por Borges e Andrade (2018) são válidas.

Transações com partes relacionadas são transações normais de mercado, mas deve haver uma atenção maior dos auditores a estas transações. A pesquisa pode contribuir para os investidores, normalizadores, auditores e pesquisadores em diversos aspectos, em que para os investidores recomenda a necessidade de atenção e a importância destas transações, enquanto que para os normalizadores e auditores há necessidade de avanços na legislação, de transparência, e meios de governanças corporativas com o intuito de inibir as transações para interesses próprios. Para os pesquisadores, fica demonstrada a importância e relevância do tema, além da necessidade de pesquisas para a compreensão completa sobre as transações com partes relacionadas. O trabalho apresenta limitações de pesquisa, associadas às variáveis, por utilizar o volume das transações, independente da obtenção anormal de ganhos e, assim, não foi possível analisar o impacto na probabilidade na ocorrência de fraude para as empresas que apresentam transações anormais com partes relacionadas, pois não há análise sobre a qualidade da informação contábil. Assim, por não estar no escopo do artigo, não se buscou identificar outros meios de se realizar fraudes. Outras limitações são devidas ao recorte temporal do estudo e, também, por só considerar as fraudes com condenações. Denúncias que estão na justiça não foram identificadas e consideradas, e sugere-se que as pesquisas futuras verifiquem se existe associação de transação com partes relacionadas e fraudes, para as outras instituições financeiras como, também, buscar identificar outros meios e oportunidades que afetam a probabilidade de ocorrência de fraude e verificar impactos dos aspectos externos (crises econômicas, políticas e pandemias) na probabilidade de ocorrência de fraude. 


\section{REFERÊNCIAS}

Abdullahi, R., \& Mansor, N. (2015). Fraud Triangle Theory and Fraud Diamond Theory. Understanding the Convergent and Divergent For Future Research. International Journal of Academic Research in Accounting. Finance and Management Sciences, v. 5, n. 4, p. 38-45. DOI: 10.6007/IJARAFMS/v5-i4/1823

Akelola, S. (2012). Fraud in the banking industry: a case study of Kenya. (Tese de Doutorado). Nottingham Trent University, Nottingham, Reino Unido. Recuperado de http://irep.ntu.ac.uk/id/eprint/147/

Alves, C., \& Alves, N. (2008). Análise do nível de divulgação da governança corporativa segundo recomendações do comitê de Basiléia: Um estudo de caso em bancos privados e públicos brasileiros. Revista Universo Contábil, 5(1), 06-21. http://dx.doi.org/10.4270/ruc.20095

Allison, P. D. (2009). Fixed Effects Regression Models. Sage. Los Angeles, USA. Vol.160. 136 p. https://dx.doi.org/10.4135/9781412993869

Berkman, H., Cole, R. A., \& Fu, L. J. (2007). Expropriation through loan guarantees to related parties: Evidence from China. Journal of Banking \& Finance. Vol. 33, 141-156. https://doi.org/10.1016/j.jbankfin.2007.11.001

Botinha, R., \& Lemes, S. (2019). Corrupção percebida: Uma Análise da Associação com o ambiente contábil dos países do G20. Advances in Scientific and Applied Accounting, 1(1), 120-139. Recuperado de https://asaa.anpcont.org.br/index.php/asaa/article/view/476

Borges, S. R. P., \& Andrade, M. E. M. C. (2018). A opinião de especialistas sobre as variáveis relacionadas ao risco de fraude contábil no setor bancário brasileiro. Revista Universo Contábil. V.14. n. 3. P. 66-88. DOI: 10.4270/ruc.2018320

Brazel, J. F., Jones, K. L., \& Zimbelman, M. F. (2009) Using Nonfinancial Measures to Assess Fraud Risk. Journal of Accounting Research, v. 47, n. 5, p. 1135-1166. https://doi.org/10.1111/j.1475-679X.2009.00349.x

Chen, Y., Zhu, \& Wang, Y. (2011). Corporate fraud and bank loans: Evidence from China. China Journal of Accounting Research. Vol. 4. 155-165. DOI: 10.1016/i.cjar.2011.07.001

Cheung, Y., Rau, R, \& Stouraitis, A. (2006). Tunneling, Propping and expropriation: evidence from connected party transactions in Hong Kong. Journal of Financial Economics 82. 343-386. https://doi.org/10.1016/i.jfineco.2004.08.012

Coenen. T. L. (2008). Essentials of Corporate Fraud. EUA: John Wiley \& Sons, Inc.

Cohen, J., Ding, Y., Lesage, C., \& Stolowy, H. (2010). Corporate Fraud and Managers' Behavior: Evidence from the Press. Journal of Business Ethics. Volume95. 271-315. DOl:10.1007/s10551-011-0857-2 
Cressey, D. R. (1953). Other people's money: a study in the social psychology of embezzlement. Glencoe, IL: The Free Press

El-Helaly, M. (2018). Related-party transactions: a review of the regulation, governance and auditing literature. Managerial Auditing Journal. Vol. 33 No. 8/9, pp. 779-806. https://doi.org/10.1108/MAJ-07-2017-1602

Fávero, L. P. L, \& Belfiore, P. (2017). Análise de dados: Modelos de regressão com Exce|®, Stata® e SPSS $\AA^{\circledR}$.1. Ed. Rio de Janeiro. Elsevier Brasil.

Firth, M., Rui, O. M., \& Wu, W. (2011). Cooking the books: Recipes and costs of falsified financial statements in China. Journal of Corporate Finance. 17, 371390. DOI: $10.1016 / j$ j.jcorpfin.2010.09.002

Friedman, E., Johnson, S., \& Mitton, T. (2003). Propping and tunneling. Journal of Comparative Economics. 31. 732-750

G1 $(2012,09,04)$. Justiça aceita denúncia contra 17 por rombo no banco Panamericano. Recuperado em 26 de outubro, 2019, de http://gl globo.com/economia/noticia/2012/09/justica-recebe-denunciacontra-17-por-rombo-no-banco-panamericano.html

G1 $(2016,20,06)$. Odebrecht comprou banco para pagar propina no exterior, diz delator. Recuperado em 27 de abril, 2020, de http://gl.globo.com/pr/parana/noticia/2016/06/odebrecht-comproubanco-para-pagar-propina-no-exterior-diz-delator.html

Giroux, G. (2008). What went Wront? Accounting Fraud and Lessons from the Recent Scandals. Social research, Volume 75, N 4, 1205-1238.

Jiang, G., Lee. C. M. C., \& Yue, H. (2010). Tunneling through intercorporate loans: The China experience. Journal of Financial Economics 98 1-20

Koholbeck, M., \& Mayhew, B. W. (2016). Are Related Party Transactions Red Flags. Contemporary Accounting Research. Volume 34. 900-928. Recuperado de https://doi.org/10.1111/1911-3846.12296

Lou, Y. I., \& Wang, M. L. (2009). Fraud Risk Factor of the Fraud Triangle Assessing the Likelihood of Fraudulent Financial Reporting. Journal of Business \& Economics Research (JBER), 7(2). https://doi.org/10.19030/jber.v7i2.2262

LU. H. R. (2017) Related Party Transactions. (Tese de doutorado em Filosofia). Rotman School of Management. University of Toronto. Canada

Machado, M. R. R. (2015). Investigação da ocorrência de fraudes corporativas em instituições bancárias brasileiras à luz do triângulo de fraude de Cressey. (Tese de doutorado em Administração). Universidade de Brasília. Brasília, DF, Brasil. http://dx.doi.org/10.26512/2015.02.T.18006 
Machado, M., \& Gartner, I. (2018). A hipótese de Cressey (1953) e a investigação da ocorrência de fraudes corporativas: uma análise empírica em instituições bancárias brasileiras. Revista Contabilidade \& Finanças, 29(76), 60-81. https://doi.org/10.1590/1808-057x201803270

Mangala, D. \& Kumari, P. (2016). Auditors' Perception of Red Flags: Indian Evidence. In Usha Arora, Deepa Mangala \& Ubba Savita (Eds.), Management Mosaic Traversing Across Assorted Research Arena (pp. 97-111). India: Wisdom Publication. https://doi.org/10.1177/0974686217738683

Oda, P. (2011). Transações com partes relacionadas, governança corporativa e desempenho: um estudo com dados em painel (Dissertação Mestrado), Faculdade de Economia, Administração e Contabilidade, Universidade de São Paulo, São Paulo, São Paulo, Brasil. DOl:10.11606/D.12.2012.tde$\underline{02052012-211106}$

OECD. (2012) Latin American corporate governance roundtable task force report related party transactions. Recuperado de https://www.oecd.org/daf/ca/LatinAmericanReportonRelatedPartyTransac tions.pdf

Oliveira, E. B. (2016). Manipulação de resultados: estudo de caso de um banco brasileiro. (Tese de Doutorado), Faculdade de Economia, Administração e Contabilidade, Universidade de São Paulo, São Paulo. DOI:10.11606/T.12.2016.tde-12072016-113525.

Pedneault. S. (2009). Fraud 101 Techniques and Strategies for Understanding Fraud. John Wiley \& Sons Inc. Third Edition

Petrucelli, J. R. (2012). Detecting Fraud in Organizations: Techniques, Tools, and Resources. Inc. John Wiley \& Sons, Inc. EUA

Pizzo. M. (2011). Related Party under a contigency perspective. Journal of Management \& Governance, Volume 17. 309330.https://doi.org/10.1007/s10997-011-9178-1

Pozzoli, M., \& Venuti, M. (2014). Related party transactions and financial performance: Is there a correlation? Empirical evidence from Italian listed companies. Open Journal of Accounting, 3(01), 28. DOI: $10.4236 /$ ojacct.2014.31004

Schuchter, A., \& Levi, M. (2014) Beyond the fraud triangle: Swiss and Austrian elite fraudsters. Accouting Forum $39 . \quad 176$ 187.http://dx.doi.org/10.1016/j.accfor.2014.12.001

Shirur, S. (2011). Tunneling vs Agency Effect: A case study of Enron and Satyam. Vikalpa. Vol. 36 No 3. 9-20. https://doi.org/10.1177/0256090920110302

Singleton, T. W., Singleton, J. A, Bologna, G. J., \& Lindquist, R. J. (2006). Fraud Auditing e Forensic Accouting. EUA. Wiley. Third Edition. 
Skousen, C. J., Smith, K. R, \& Wright, C. J. (2009). Detecting and predicting financial statement fraud: the effectiveness of the fraud triangle and SAS no. 99. Corporate governance and firm performance advance in financial economics. Vol. 13. 53-81. DOI: $10.1108 / S 1569-3732(2009) 0000013005$

Supatmi, S. T., Saraswati E., \& Purnomosidhi, B. (2019). The effect of related party transactions on firm performance: the moderating role of political connection in indonesian banking. Business: Theory and Practice 20: 81-92. https://doi.org/10.3846/btp.2019.08

Thomas, W. C. (2002). The Rise and Fall of Enron. Journal of Accountancy. 193: $41-$ 48.

Umobong, A. A. (2017). Related Party Transactions and Firms Financial Performance. African Research Review 11 (1):60. DOI: 10.4314/afrrev.v1 1i1.5

Wells, J. T. (201 1). Financial Statement Fraud Case book Baking the ledgers and cooking the books. ACFE. First Edition.

\section{CONTRIBUIÇÕES DOS AUTORES}

\begin{tabular}{|l|c|c|c|c|}
\hline \multicolumn{1}{|c|}{ Contribuição } & $\begin{array}{c}\text { Júlio César } \\
\text { Gomes } \\
\text { Mendonça }\end{array}$ & $\begin{array}{c}\text { Michele Rílany } \\
\text { Rodrigues } \\
\text { Machado }\end{array}$ & $\begin{array}{c}\text { Ercilio } \\
\text { Zanolla }\end{array}$ & $\begin{array}{c}\text { José Alves } \\
\text { Dantas }\end{array}$ \\
\hline $\begin{array}{l}\text { 1. Idealização e concepção do } \\
\text { assunto e tema da pesquisa }\end{array}$ & $\checkmark$ & $\checkmark$ & & \\
\hline $\begin{array}{l}\text { 2. Definição do problema de } \\
\text { pesquisa }\end{array}$ & $\checkmark$ & $\checkmark$ & & \\
\hline $\begin{array}{l}\text { 3. Desenvolvimento da } \\
\text { Plataforma Teórica }\end{array}$ & $\checkmark$ & $\checkmark$ & & \\
\hline $\begin{array}{l}\text { 4. Delineamento da abordagem } \\
\text { metodológica da pesquisa }\end{array}$ & $\checkmark$ & $\checkmark$ & & $\checkmark$ \\
\hline 5. Coleta de dados & $\checkmark$ & & & \\
\hline $\begin{array}{l}\text { 6. Análises e interpretações dos } \\
\text { dados coletados }\end{array}$ & $\checkmark$ & $\checkmark$ & & $\checkmark$ \\
\hline 7. Conclusões da pesquisa & $\checkmark$ & $\checkmark$ & $\checkmark$ & $\checkmark$ \\
\hline 8. Revisão crítica do manuscrito & $\checkmark$ & $\checkmark$ & $\checkmark$ & $\checkmark$ \\
\hline $\begin{array}{l}\text { 9. Redação final do manuscrito, } \\
\text { conforme as normas } \\
\text { estabelecidas pela Revista. }\end{array}$ & $\checkmark$ & $\checkmark$ & $\checkmark$ & \\
\hline 10. Orientação & & $\checkmark$ & & \\
\hline
\end{tabular}

